\title{
Trabajo en producción de comidas: consecuencias en la alimentación y estado nutricional de los trabajadores
}

\author{
Work in production of meals: consequences in the \\ feeding and nutritional status of the workers
}

\author{
Matos, Cristina Henschel \\ Licenciada en Nutrición. Profesora del Curso de Nutrición. \\ Universidad del Vale do Itajaí. Itajaí. Brasil. \\ Proença, Rossana Pacheco Da Costa \\ Licenciada en Nutrición. Profesora del Departamento de Nutrición e Coordinadora \\ del Núcleo de Pesquisa de Nutrição em Producción de Comidas - NUPPRE. \\ Universidad Federal de Santa Catarina. Florianópolis. Brasil. \\ Costa, Soraya Pacheco Da \\ Diplomada en Fisioterapia. Licenciada en Documentación. \\ Profesora del Departamento de Fisioterapia. Universidad de Alcalá. \\ Correspondencia: \\ Cristina Henschel Matos \\ Departamento de Nutrição, Campus Universitário- UFSC. \\ 88040-900 - Florianópolis, SC, BRASIL \\ cmatos@univali.br
}

Fuente de Financiación: Fundo de Amparo à Pesquisa da Universidade

Federal de Santa Catarina - FUNPESQUISA y Instituto DANONE - BRASIL

Resumen

El objetivo del presente estudio es identificar la relación entre trabajar en la producción de comidas y la alimentación y estado nutricional de trabajadores que desarrollan su labor en ese sector. Se trata de una investigación cualitativa y descriptiva desarrollada como estudio de caso, utilizando el abordaje del Análisis Ergonómico del Trabajo. La recopilación de datos se realizó a través de entrevistas con trabajadores y observación directa de actividades desarrolladas en el puesto de trabajo. Además, se recogieron datos sobre el Índice de Masa Corporal, el riesgo de enfermedad metabólica asociada a la obesidad, el gasto energético y el consumo de comida durante el trabajo. Los resultados demuestran que la mayoría de los trabajadores presenta un gasto energético significativo en relación a las actividades que realizaban durante su turno de trabajo. Sin embargo, en la evaluación del estado nutricional se pudo evidenciar que solamente dos trabajadores presentaran eutrofia mientras los demás presentaran exceso de peso. El tipo de comida que era consumida por la mayoría de los trabajadores se caracteriza por ser hiperlipídica e hipoglicídica. Además, las personas estudiadas tenían el hábito de picar entre horas, probablemente debido a que, por un lado, pasaba demasiado tiempo entre una comida y la siguiente y por el otro, estaban constantemente en contacto directo con los alimentos preparados. Por ello, se ha podido evidenciar que, en este caso, el lugar de trabajo y sus características representan una fuerte influencia en el estado nutricional y el tipo de alimentación de los trabajadores estudiados.

Palabras claves: restauración colectiva, estado nutricional, trabajo, Med Segur Trab 2009; 55 (214): 91-100 


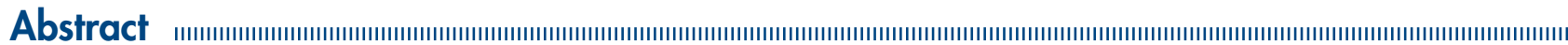

The present study aimed to identify the relationship between the nature of work with food production and eating habits, as well as the nutritional status of operators. This qualitative and descriptive research was developed as a case study, with the use of Work Ergonomic Analysis. Data was collected through interviews with the operators, and direct and equipped observation of the activities carried out during their work. Body mass index was also assessed, along with the risk of metabolic disease associated with obesity, as well as energy expenditure and food intake during work shifts. The results show that most operators had highenergy expenditure due to the activities carried out during their work shifts. However, the nutritional status assessment showed that only two operators were eutrophic and the others were overweight. Food intake was characterized by hyperlipidic and hyperglycemic diets. The operators reported regular food intake during the intervals between meals, possibly because of the long time gap between them and the direct contact with the meals they serve. In conclusion, it was observed that the place of work and its characteristics highly influenced nutritional status and eating practices of the operators.

Index terms: food service, nutritional status, work, Med Segur Trab 2009; 55 (214): 91-100 


\section{INTRODUCCIÓN}

El comportamiento alimentar de un individuo representa uno de los principales condicionantes de su estado nutricional, en relación con todas las prácticas de su alimentación ${ }^{1}$. Aunque los hábitos de alimentación se formen en la infancia, factores como el lugar de trabajo, la disponibilidad y la posibilidad de libre consumo de alimentos pueden influenciar en la práctica alimentar y, consecuentemente, alterar el estado nutricional de las personas ${ }^{2}$.

De esta manera, las unidades productoras de comidas, ya sean colectivas o comerciales, son el lugar de trabajo en los que los trabajadores están constantemente en contacto con los alimentos, y los consumen durante su jornada de trabajo, aunque esta práctica no esté permitida oficialmente.

Cabe resaltar que la mayoría de los estudios sobre el cuidado nutricional en base a la composición de las comidas, consideran solamente los comensales que utilizan dichos servicios $^{3,4,5,6,7}$. Por tanto, son pocas las referencias basadas en recomendaciones específicas a los consumidores internos, es decir, los trabajadores de una unidad de producción de comidas (UPC). Sin embargo, algunos estudios de autores brasileños demuestran un alto porcentaje de exceso de peso en dicha población y evidencian su relación con la actividad desarrollada ${ }^{8,9}$.

Además, el trabajo en la producción de comidas en Brasil está caracterizado por la realización continua de movimientos repetitivos, la carga excesiva de peso, por la larga permanencia en la postura de bipedestación y por la constante modificación de los procedimientos de trabajo, lo que resulta en un significativo gasto energético durante la jornada de trabajo ${ }^{10}$.

Un estudio reciente sugiere que un alto porcentaje de enfermedades venosas están presentes en los trabajadores de UPC y que este hecho puede estar relacionado con las condiciones de trabajo en conjunto con el sobrepeso de la mayoría de los trabajadores ${ }^{11}$.

Consecuentemente, el sector de producción de comidas empieza a preocuparse con la salud del trabajador, en la medida en que aumenta la percepción de la influencia de las prácticas alimentares y de la salud en el desempeño y productividad en el trabajo. Debido a todo lo expuesto, el presente estudio tiene el objetivo de identificar la relación entre el trabajo en la producción de comidas en relación con la práctica alimentar y el estado nutricional de los trabajadores.

\section{METODOLOGÍA}

Este es un estudio descriptivo, desarrollado como estudio de caso en el que se utilizó el abordaje del Análisis Ergonómico del Trabajo (AET) ${ }^{12}$, además de incluir la valoración del estado nutricional de los trabajadores, realizado a través de las medidas antropométricas y del consumo alimentario. El AET es un método de evaluación basado en la comparación entre las condiciones disponibles para la realización de la tarea y la manera en la que el trabajo es realizado. Por ello, la riqueza de este método radica en la posibilidad de observación del ser humano en actividad, es decir, de como el trabajo se desarrolla y cuales son los factores que interfieren tanto positiva como negativamente en esa situación ${ }^{12}$.

La investigación se realizó en una empresa ubicada al sur de Brasil, en una ciudad colonizada por Alemanes. Esta empresa funciona durante 24 horas, sus trabajadores realizan turnos de 6 horas diarias y cada trabajador recibe una comida al día. La UPC elabora 4 comidas al día y, en total, sirve aproximadamente 1550 comidas cada día. Más específicamente, en la UPC trabajan 24 personas divididas en 4 turnos, de forma que 10 trabajadores están en el primer turno (desde las 5.00 hasta las 14.18 horas), 3 están en el turno general (desde las 7.30 hasta las 16.48 horas), 8 están en el segundo turno (desde las 14.18 hasta las 23.24 horas) y 3 están el en tercer turno (desde las 22.00 hasta las 5.00 horas). Los trabajadores, a excepción del cocinero, están ubicados en los sectores según 
las necesidades de trabajo durante la producción. El reparto de tareas y el número de trabajadores por cada sector están determinados por los siguientes factores: tipo de carta, cantidad de comida para ser preparadas y número de trabajadores presentes.

El procedimiento de reconocimiento de la unidad se hizo a través de visitas, lecturas de documentos y entrevistas junto con la Licenciada en Nutrición responsable por la unidad. También se realizó observación directa con apoyo de instrumentos tales como grabadora, cámara de fotos y cronómetro. Para la determinación del espacio físico y la medición de los utensilios y equipamientos, se utilizó una cinta métrica y un metro electrónico, y se elaboró una figura de cada uno de los sectores de la UPC.

Los criterios de inclusión de los trabajadores fueron: desarrollar su labor en los sectores productivos de la UPC en el primer turno y en el turno general, aceptar participar en el estudio y contestar al registro de alimentación. La población que cumplía dichos criterios fue de 7 personas estudiadas.

La observación se realizó por el mismo examinador, durante la jornada de trabajo, utilizando una Guía de Observación de las Condiciones de Trabajo, con el objetivo de identificar los riesgos de accidente, la postura, los esfuerzos realizados y el ambiente térmico, luminoso y sonoro. Los niveles de ruido se verificaron a través de un medidor de nivel de presión sonora (marca LUTRON ${ }^{\circledR}$ ) en los siete principales sectores de la UPC, el espacio físico y la medida de los utensilios se hizo con una cinta métrica y metro electrónico (marca ZIRION ${ }^{\circledR}$ ). Además, se realizaron entrevistas y se aplicaron cuestionarios a los trabajadores que participaran en el estudio.

La verificación de las medidas antropométricas de los trabajadores se hizo a partir de las variables peso, estatura corporal y circunferencia abdominal (medida a la altura del ombligo, sin comprimir los tejidos) ${ }^{13}$.

Para valorar el estado nutricional se utilizó el Índice de Masa Corporal (IMC), correspondiente a peso dividido por estatura al cuadrado, según los puntos de corte de la Organización Mundial de la Salud (OMS) ${ }^{14}$ mientras que para la valoración del riesgo de enfermedades metabólicas asociadas a la obesidad, se midió la circunferencia abdominal según los puntos de corte propuestos por Han et al. ${ }^{13}$

Para determinar el gasto energético de los trabajadores se utilizó la fórmula propuesta por el Institute of Medicine (IOM) ${ }^{15}$ que considera las variables sexo, edad, actividad física (trabajo y ocio), estatura (e) y peso teórico $\left(q=\mathrm{e}^{2} / 21,5\right.$ y $\left.\hat{\delta}=\mathrm{e}^{2} / 22,5\right)$.

En el desarrollo del estudio, se procedió a la valoración del consumo de alimentación de los trabajadores por un periodo de tres días alternos de trabajo, con la aplicación del recordatorio de alimentación por el investigador y del registro diario de dos días apuntados por el trabajador, ambos con la ayuda de un libro con el registro fotográfico de los utensilios y las raciones de alimentos. Los resultados de la ingesta de calorías y de los macronutrientes fueran representados por la media de los tres días de la semana y comparados con las recomendaciones del $\operatorname{IOM}^{15}$ y de la OMS $^{16}$ respectivamente. Además se realizó el acompañamiento de todas las comidas realizadas en el lugar de trabajo, donde se observó el horario y el tiempo de las mismas, la elección de los alimentos y de la compañía para comer.

Para la comparación entre las condiciones de trabajo y el estado nutricional se hizo un examen, una categorización y una tabulación de las evidencias encontradas. El estudio fue sometido y aprobado por el Comité de Ética en la investigación con Seres Humanos de la Universidad Federal de Santa Catarina.

\section{RESULTADOS}

Como se comentó anterioremente, la UPC analizada está en una empresa que funciona durante 24 horas con 4 turnos de 6 horas diarias, de forma que cada trabajador recibe una "gran comida" al día. La carta consta de dos tipos de carnes, guarnición, arroz, frijoles, tres 
tipos de ensalada, pan, dos tipos de salsa para la ensalada y dos tipos de bebidas (té y zumo artificial endulzado). El sistema de distribución es a través de buffet libre con una limitación de un tipo de carne por persona. Sin embargo, los trabajadores de la UPC no tienen ningún tipo de limitación en cuanto a la variedad y cantidad de carne.

De los siete trabajadores que participaron en el estudio, seis son mujeres y uno es hombre (Número 1. Figura 1). El aumento de peso, el tiempo de trabajo en la unidad, el IMC y la circunferencia abdominal están representados en la Figura 1.

Figura 1. Sector de actuación, tiempo de trabajo, aumento de peso y diagnóstico nutricional de los trabajadores analizados.

\begin{tabular}{rlcccc}
\hline Operador & Sector de actuación principal & $\begin{array}{c}\text { Tiempo de } \\
\text { servicio en la } \\
\text { UPC (meses) }\end{array}$ & $\begin{array}{c}\text { Aumento de } \\
\text { peso (kg) }\end{array}$ & $\begin{array}{c}\text { IMC } \\
(\mathbf{k g} / \mathbf{m} 2)\end{array}$ & $\begin{array}{c}\text { Circunferencia } \\
\text { abdominal (cm) }\end{array}$ \\
\hline 1 & Recepción y almacenaje & 45 & 0 & 19,36 & 77 \\
2 & Pré-preparación de veeduras & 60 & 5,5 & 24,3 & 85 \\
3 & Higiene de bandejas & 66 & 5 & 25,3 & 84 \\
4 & Higiene de cazos & 4 & 9 & 26,9 & 100 \\
5 & Cocción & 60 & 9,5 & 28,7 & 85 \\
6 & Higiene de bandejas & 3 & 7 & 31,25 & 100 \\
7 & Distribución & 66 & 3 & 33,3 & 87 \\
\hline
\end{tabular}

El IMC indicó una eutrofia en dos de los trabajadores y sobrepeso, en diferentes grados, en cinco de los trabajadores. La medida de la circunferencia abdominal pudo demostrar que todos los trabajadores clasificados con sobrepeso presentaban riesgo, aumentado o muy aumentado, para el desarrollo de enfermedades metabólicas asociadas con la obesidad13.

Por medio de observación directa y de los niveles de actividades establecidos pela IOM $^{15}$ fue posible constatar que, de la totalidad de los trabajadores estudiados, las seis del sexo femenino fueron clasificadas como activas e el trabajador del sexo masculino fue clasificado como poco activo. La Necesidad Energética Total (NET) media de los trabajadores fue de $2236 \pm 142 \mathrm{kcal}$, mientras que la media de consumo fue de $2400 \pm 409 \mathrm{kcal}$.

La dieta consumida por los trabajadores se define como hiperprotéica e hipoglicídica. Estos datos están representados en la Figura 2.

Figura 2. Recomendaciones de macronutrientes FAO/WHO (2003) comparadas con la ingesta de los trabajadores estudiados.
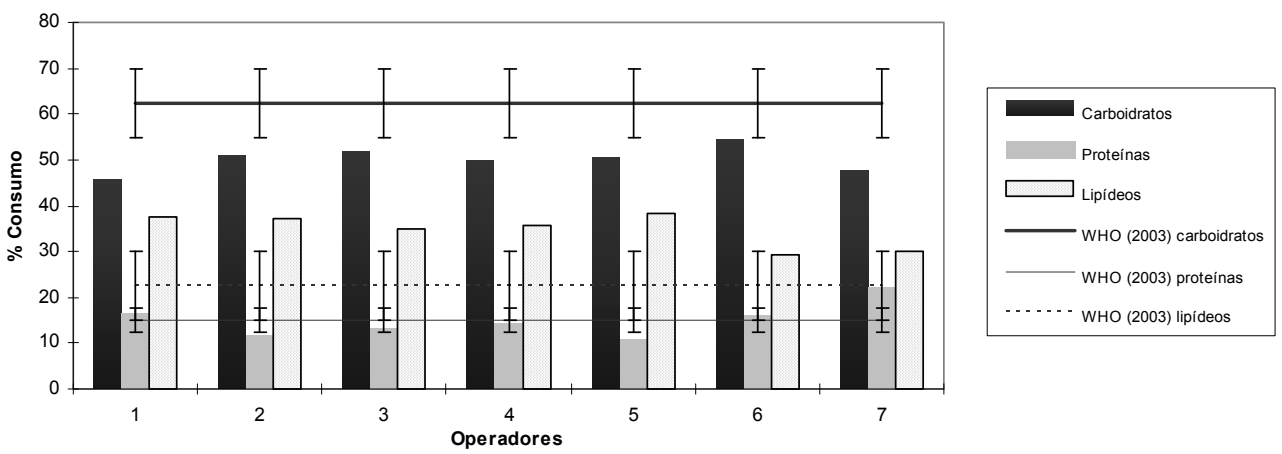

En relación a las características ambientales, el espacio físico total da UPC fue considerado satisfactorio, a excepción de los sectores de almacenaje, higienización de las bandejas y cocción, que presentan un espacio reducido para la circulación de los carros 
de transporte. En consecuencia los trabajadores son demandados de un esfuerzo extra para el manejo de las cargas de trabajo.

La UPC presenta problemas de ruido, temperatura y humedad. Los sectores de cocción e higiene de bandejas son los más afectados por los efectos térmicos (temperatura, humedad y ventilación), debido a la irradiación de calor de los respectivos equipamientos, tales como, freidora, fuegos de la cocina y el lavavajillas. Incluso se observó la necesidad de que los trabajadores tuviesen que secar el techo hasta dos veces en cada turno de trabajo, de forma que adoptaban posturas inadecuadas, con el consecuente aumento del gasto energético. Precisamente en los trabajadores de esos dos sectores donde se constató una frecuencia más alta de consumo de bebidas calóricas, puesto que el consumo de agua no es abundante, prevaleciendo la ingesta de bebidas protéicas y/o edulcoradas.

El nivel de ruido constante proviene de la campana extractora y del lavavajillas, mientras que los ruidos ocasionales provienen tanto de los equipamientos utilizados durante un corto espacio de tiempo, tanto por el picador de verduras, la batidora y la señal sonora del horno, como por la conversaciones o el choque de los utensilios metálicos. Dichos ruidos dificultan la comunicación entre los trabajadores y hace que los mismos se tenga que desplazar constantemente para en desarrollo satisfactorio de las actividades.

Los movimientos repetitivos se observaron durante el corte de verduras y para freir algunos alimentos, ya que el trabajador tenía que permanecer en bipedestación estática con el cuello flexionado durante un largo periodo de tiempo.

Debido a que las actividades del sector de cocción son consideradas prioritarias, se pudo observar que el aumento o disminución del número de trabajadores ubicados en este sector dependía del menú de cada día. Además, también se pudo verificar que el ritmo de las actividades realizadas variaba según la cantidad de trabajo y el tiempo que quedaba para el final de la jornada, por ejemplo, en los días en que había fritos en el menú, las actividades tenían que ser hechas más rápidamente, debido a que este tipo de preparación demanda más tiempo.

La ubicación de los trabajadores también tuvo influencia en los horarios de comida, debido a que si el trabajador estuviese ubicado en el sector de higiene de equipamientos, tenía que comer hasta dos horas antes de los demás, normalmente solo y preocupado con a realización de la actividad que tenía que realizar después.

Debido a que esta unidad sirve grandes comidas en todos los turnos, aparece la disponibilidad de carnes y fritos durante toda la jornada de trabajo. Por tanto, aún durante el almuerzo, todos os trabajadores optaron por el consumo de carne, generalmente grasa, o que contribuye significativamente para la valoración del tipo de dieta ingerida a lo largo del día.

\section{DISCUSIÓN}

Aunque el espacio físico en la UPC sea considerado satisfactorio, los problemas relacionados con el espacio de circulación en los sectores de cocción, almacenaje e higiene de bandejas e utensilios implican un esfuerzo físico importante por parte de los trabajadores en las tareas de levantar y cargar peso.

En el sector de cocción, además de problemas relacionados con la circulación, habían un número insuficiente de encimeras, lo que hace que algunas actividades se realicen en posturas inadecuadas, lo que puede provocar dolores de espalda o aumentarlos en caso de que ya existan.

Resultados similares fueron encontrados en un estudio realizado con cocineras de comedores escolares, donde las deficiencias de estructura física, principalmente en relación a la altura de las encimeras y mesas de apoyo, ocasionaban esfuerzos y posturas inadecuadas en una población de la que el $46 \%$ de los trabajadores presentaba problemas de columna y el $47 \%$ relataba dores de espalda ${ }^{17}$. 
La incomodidad térmica descrita por los trabajadores en los sectores de cocción, higiene de bandejas, cazos y otros utensilios, convierte el trabajo en una actividad más desgastante y aumenta la posibilidad de que los trabajadores se equivoquen. Además, el calor produce una sudoración excesiva, que debe ser compensada por una rehidratación regular ${ }^{18}$. De esta manera, el alto consumo de líquidos por los trabajadores de dichos sectores refleja la adaptación al calor. Sin embargo, se pudo constatar que el consumo de agua es prácticamente nulo, ya que la mayoría de los trabajadores que tienen sed, recurren a zumos edulcorados (159kcal/ 200ml) o a bebidas proteicas, como el café con leche entera.

Niveles de ruido por encima de lo recomendado por la $\mathrm{NR} 15^{19}$, es decir $85 \mathrm{~dB}$ (A) por 8 horas, fueron encontrados en los sectores de cocción e higiene de bandejas. El sector de higiene de cazos presentó picos de ruido de hasta $95 \mathrm{~dB}$ (A) cuando se utilizaba la batidora. El ruido excesivo dificulta la comunicación entre los trabajadores, y por ello se tienen que desplazar a otros sectores para obtener información sobre algo que necesiten.

Las condiciones de trabajo identificadas, además de ser algunos de los factores responsables por el estrés en el trabajo, aumentan la exigencia de esfuerzo físico por parte de los trabajadores con el consecuente gasto energético ${ }^{20}$.

Durante el período de seguimiento del trabajo y de las comidas, se pudo constatar que el horario de entrada y salida se cumplían rigurosamente, mientras que el horario de las comidas variaban según el ritmo de las actividades diarias. Como había intervalos de hasta 5 horas entre las comidas, el apetito de los trabajadores aumentaba, lo que les llevaba a consumir alimentos hipercalóricos entre horas.

El horario de las comidas pode afectar el peso corporal, ya que la respuesta termogénica (aumento del metabolismo estimulado por la ingesta de alimentos) es significativamente mayor en una dieta consumida durante la mañana, que en una dieta consumida durante la tarde o noche ${ }^{21}$. Además, la distribución de tres comidas al día puede provocar un aumento de peso más alto que en un mayor número de veces al día ${ }^{22}$.

El hecho más preocupante de la situación estudiada, a parte de la cantidad de alimentos ingeridos, es la calidad de la alimentación consumida por los trabajadores, tanto en el almuerzo como en la comida. En la comparación entre la ingesta de calorías de los trabajadores estudiados con las Necesidades Energéticas Totales (NET) recomendadas por la $\mathrm{IOM}^{15}$, se pudo verificar que de los siete trabajadores estudiados, cuatro consumen por encima de sus necesidades energéticas durante los días de trabajo, uno consume lo que necesita y precisamente los dos que relataron consumir menos que el NET, son aquellos que presentaban un IMC por encima de $30 \mathrm{~kg} / \mathrm{m}^{2}$ y que trabajan en los sectores que exigen más desplazamiento. El relato de ingesta de estos individuos puede pode estar subestimado por la preocupación con el peso actual, no obstante, ninguno de los trabajadores mencionó estar haciendo restricciones en cuanto a su alimentación durante el periodo del estudio.

Este dato caracteriza claramente dos de las limitaciones de los métodos de valoración de consumo alimentario: la omisión de alimentos consumidos con poca frecuencia y la dificultad de los individuos en estimar y cuantificar los alimentos consumidos ${ }^{23}$. Otro factor importante es la subnotificación de la ingesta energética y su asociación con la obesidad y con las características psicosociales como la restricción dietética y el deseo de ajuste social ${ }^{24}$.

En relación al consumo de alimentos ricos en proteínas, el 57\% de los trabajadores presentan una ingesta media de proteínas entre el 10\% y el 15\%, según las recomendaciones de la $\mathrm{OMS}^{16}$, lo que demuestra una alimentación hiperproteica para los demás trabajadores $(57,14 \%)$. La causa más probable de ese desequilibrio en la ingesta se debe al hecho de que los trabajadores da UPC pueden consumir carne libremente. A ese dato, se añade que el consumo de café con leche también estaá liberado y es una costumbre bastante común entre los trabajadores.

Otro macronutriente consumido en exceso por el $85,70 \%$ de los trabajadores son los lípidos. La frecuencia de fritos y carnes grasas en el menú puede ser el responsable de ese hecho, ya que el consumo de dichos alimentos no está presente solamente durante la comida, sino también durante el almuerzo y mientras ejecutan distintas actividades. 
Es importante recordar que el potencial de una dieta o de un alimento en concreto, para aumentar los niveles de colesterol sérico y provocar aterosclerosis está directamente relacionado a su contenido de colesterol y de grasas saturadas. Algunos estudios evidencian una alta correlación entre la incidencia de enfermedades de aterosclerosis, niveles de lípidos séricos y hábitos alimentarios ${ }^{25}$.

Por otro lado, el consumo de glúcidos por todos los trabajadores era reducido, siendo inferior a lo recomendado pela $\mathrm{OMS}^{16}$, es decir, del 55\% al 70\% del total de la dieta. Probablemente ese nutriente aparece con un porcentaje reducido debido a que una dieta rica en lípidos y proteínas provoca un nivel alto de saciedad.

Cabe resaltar que, aunque el menú ofertado por la empresa no haya sido exhaustivamente analizado, cuando es consumido en las cantidades sugeridas por un Nutricionista, este debe ser equilibrado en el sistema de distribución para los comensales externos. Sin embargo, el sistema de distribución de comidas adoptado por los trabajadores de la UPC estudiada es el buffet libre, lo que permite al comensal elegir cualquiera de la preparaciones sin límite de cantidad. Considerándose que el aumento de la posibilidad de elección es un fenómeno contemporáneo que afecta también a la alimentación, probablemente la cuestión no sea la libre oferta, sino que las condiciones que llevan a los trabajadores a comer exageradamente.

Otro punto que demuestra la influencia del local de trabajo en las prácticas alimentares es el hecho de que todos los trabajadores, al permanecer durante la mayor parte del día en la unidad, realizan la mayoría de sus comidas en el local de trabajo, de forma que apenas se alimentan en sus casas. Los salarios bajos, la distancia de casa al trabajo y la mayor disponibilidad y variedad de alimentos en su local de trabajo puede que justifiquen esta evidencia.

A través de la observación directa y de la evaluación de las prácticas alimentares, es posible concluir que la alimentación de los trabajadores es mayoritariamente hiperlipídica e hipoglicídica y que, a pesar de que mencionaban el aumento del consumo de frutas y verduras, tras haber empezado a trabajar en la UPC, la mayoría de los trabajadores tuvo su peso corporal aumentado.

Como consecuencia de las condiciones anteriormente descritas, se pudo observar que, aunque la actividad ocupacional esté clasificada como activa para la mayoría de los trabajadores, el IMC apunta que el $71,4 \%(n=5)$ presentan exceso de peso.

Un estudio realizado con trabajadores de UPC en hospitales en el sur de Brasil, demuestra la prevalencia de exceso de peso $(71,5 \%$; $=143)$, y que la variable "picar entre horas", era una condición específica de este tipo de trabajador ${ }^{8}$. Resultados similares se encontraron en la evaluación de 48 trabajadores de una UPC, de los cuales, el 68,75\% $(n=33)$ presentaban exceso de peso, y el consumo de frutas y verduras era insuficiente para el $77,08 \%$ y el $87,5 \%$ de los trabajadores respectivamente ${ }^{9}$

No obstante, pese a los problemas físicos y ambientales encontrados en la UPC, la mayoría de los trabajadores (80\%) se sienten motivados con su trabajo. Parce que este hecho se debe a las condiciones de organización de la unidad, especialmente en la relación cordial que se mantiene con la dirección. La participación en las decisiones y una autonomía relativa en la ejecución de sus funciones, contribuyen para la motivación de los trabajadores.

Otro hecho relacionado con la satisfacción mencionado por los trabajadores, es el poder comer durante toda a jornada de trabajo, incluso entre las comidas.

\section{CONSIDERACIONES FINALES}

Los datos analizados en esta investigación demuestran que, por un lado, las actividades de los trabajadores en la UPC requieren un gasto energético significativo, por 
otro, las prácticas alimentares incorrectas y una dieta nutricionalmente desequilibrada se reflejan en el estado nutricional de los trabajadores.

En relación a las condiciones físicas y ambientales de la situación estudiada, se puede ver que éstas influyen en el aumento del gasto energético de los trabajadores, alterando claramente el consumo alimentar. No obstante, el estado nutricional de los trabajadores no es acorde con el gasto energético elevado exigido para la realización de las tareas en la UPC. Este hecho, se justifica, probablemente pela propia naturaleza del trabajo y de las condiciones de organización, es decir, el contacto directo con la alimentación, la disponibilidad de alimentos y preparados grasos, la ausencia de criterios en la elección de los alimentos consumidos y la posibilidad de su consumo de forma indiscriminada, estarían favoreciendo los índices de sobrepeso encontrados. Además, cabe resaltar que el estrés generado por las condiciones físicas y organizativas adversas pueden estar contribuyendo para el aumento de la ingesta alimentar.

Entre las principales medidas recomendadas están: alternar las actividades de los trabajadores para evitar la ejecución de trabajos repetitivos por largos períodos de tiempo; reestructurar el puesto de trabajo del operador del sector de higiene; implantar un programa de gimnasia laboral, preparatoria y compensatoria; reestructurar el espacio físico destinado al sector de almacenaje, respetando los espacios de circulación y altura máxima de almacenaje; redistribuir los horarios de las comidas e incentivar el consumo de agua fría, principalmente en verano.

\section{REFERENCIAS BIBLIOGRÁFICAS}

1. Assis MAA, Kupek E, Nahas M V, Bellisle F. Food intake and circadian rhythms in shift workers with a high work load. Appetite, 2003; (40)2:175-183

2. Matos CH. A influencia das condições de trabalho no estado nutricional de trabalhadores do setor de alimentação coletiva: um estudo de caso. 2000. 133 f. Dissertação (Mestrado em Engenharia de Produção)Programa de Pós-Graduação em Engenharia de Produção/Ergonomia, Universidade Federal de Santa Catarina, Florianópolis. Disponible en: <http://teses.eps.ufsc.br/Resumo.asp?1075>.

3. Savio KEO, Costa, THM Miazaki E, Schmitz BAS. Avaliação do almoço servido a participantes do programa de alimentação do trabalhador. Rev Saúde Pública 2005; 39: 145-55.

4. Glanz K, Hoelscher D. Increasing fruit and vegetable intake by changing environments, policy and pricing: restaurant-based research, strategies, and recommendations. Prev Med. 2004; 39: S88-S93.

5. Nielsen SJ, Popkin BM. Patterns and trends in food portion sizes, 1977-1998, JAMA 2003; 289: 450-453.

6. Guthrie SJ; Lin BH; Frazao E., Role of food prepared away from home in the American diet, 1977-78 versus 1994-96: changes and consequences. J Nutr Educ Behav. 2002; 34: 140-150

7. Composition des repas servis en restauration scolaire et sécurite des aliments. Cah. Nutr. Diét. 2001, 36(5): 348-354.

8. Boclin KLS; Blanck N. Excesso de peso: características de trabalhadores do setor de alimentação coletiva. RBSO. 2006; 31(113):41-47.

9. Höfelmann DA, Rieke B.H, Azevedo LC. Hábito alimentar e estado nutricional: fatores de risco cardiovascular entre colaboradores internos de uma Unidade de Alimentação e Nutrição. Nutrição em Pauta. 2005; 18(70): $42-45$.

10. Matos CH, Proença RPC. Condições de trabalho e estado nutricional de trabalhadores do setor de alimentação coletiva: um estudo de caso. Brazilian Journal of Nutrition. 2003; 16(4):493-502.

11. Bertoldi CML, Proença RPC, GGN, Costa SP Condiciones de trabajo en la producción de comidas como factores de riesgo para la enfermedad venosa de miembros inferiores. Med. segur. trab. 2007; 52(206): 25-32

12. Wisner A. Por dentro do trabalho : ergonomia, método \& técnica. São Paulo: FTD; 1987.

13. Han TS, Van Leer EM, Seidell JC, Lean MEJ. Waist circumference a level in the identification of cardiovascular risk factors: prevalence study in a random sample. BMJ. 1995; 311:1401-05.

14. WORLD HEALTH ORGANIZATION. Obesity preventy and managing the global Epidemic. Geneve: WHO, 1998.

15. INSTITUTE OF MEDICINE - IOM. Dietary Reference Intakes for Energy, Carbohydrate, Fiber, Fat, Fatty Acids, Cholesterol, Protein, and Amino Acids. Washington (DC); 2002.

16. WORLD HEALTH ORGANIZATION - WHO. Diet, nutrition and prevention of chronic diseases. Geneva; 2003. 
17. Proença RPC, Matos CH. Condições de trabalho e saúde na produção de refeições em creches municipais de Florianópolis. Rev. Ciências da Saúde. 1996; 15(12): 73-84.

18. Frache, B. Le risques professonnels en restauration collective. Clichy: BPI, 2000.

19. Ministério Do Trabalho. Normas Regulamentadoras do Trabalho. 2005. Disponible en: <http://www.mtb.gov.br/ >.

20. Grandjean E. Manual de ergonomia, adaptando o trabalho ao homem. 4ed, Porto Alegre: Bookman;1998.

21. Castro JM. The time of day of food intake influences overall intake in humans. J Nutr. 2004; 134(1):104-11.

22. Gauche H, Calvo MCM, Assis MAA de. Ritmos circadianos de consumo alimentar nos lanches e comidas de adultos: aplicação do semanário alimentar. Brazilian Journal of Nutrition. 2006; 19(2): 177-185.

23. Poulain JP, Proença RPC. Reflexões metodológicas para o estudo das práticas alimentares. Brazilian Journal of Nutrition. 2003; 16(4): 365-386.

24. Scagliusi FB, Lancha Junior AH. Subnotificação da ingestão energética na avaliação do consumo alimentar. Brazilian Journal of Nutrition. 2003; 16(4): 471-481.

25. Fornés, NS. et al. Escores de consumo alimentar e niveis de lipêmicos na população de São Paulo, Brasil. Journal of Public Health. 2002; (36)1:12-18.

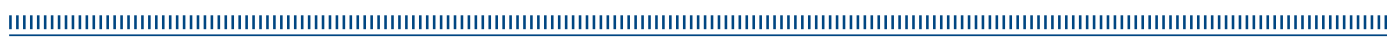

